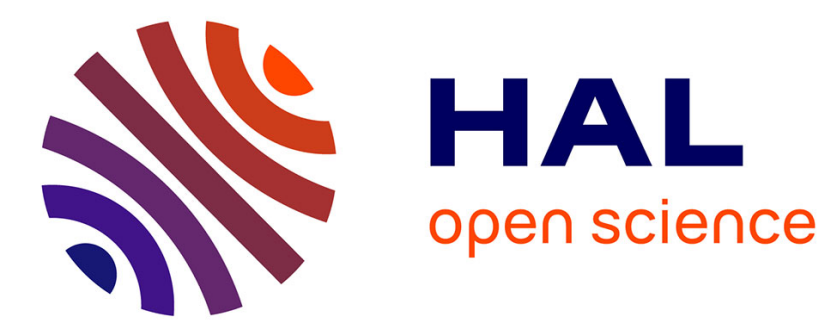

\title{
A short and versatile route to chiral spiroketal skeletons
}

\author{
A. Tursun, I. Canet, Bettina Aboab, M.-E. Sinibaldi
}

\section{To cite this version:}

A. Tursun, I. Canet, Bettina Aboab, M.-E. Sinibaldi. A short and versatile route to chiral spiroketal skeletons. Tetrahedron Letters, 2005, 46, pp.2291-2294. 10.1016/j.tetlet.2005.02.009 . hal-00125821

\section{HAL Id: hal-00125821 \\ https://hal.science/hal-00125821}

Submitted on 5 Mar 2007

HAL is a multi-disciplinary open access archive for the deposit and dissemination of scientific research documents, whether they are published or not. The documents may come from teaching and research institutions in France or abroad, or from public or private research centers.
L'archive ouverte pluridisciplinaire HAL, est destinée au dépôt et à la diffusion de documents scientifiques de niveau recherche, publiés ou non, émanant des établissements d'enseignement et de recherche français ou étrangers, des laboratoires publics ou privés. 


\title{
A short and versatile route to chiral spiroketal skeletons
}

\author{
Ahmatjan Tursun, Isabelle Canet, ${ }^{*}$ Bettina Aboab and Marie-Eve Sinibaldi*
}

Laboratoire de Synthèse Et Etude de Systèmes à Intérêt Biologique (SEESIB), UMR CNRS 6504,

Université BlaisePascal, 63177 Aubière Cedex, France

\begin{abstract}
Different chiral spiroketal skeletons are obtained, in a versatile manner, by iterative alkylations of acetone $N, N$-dimethylhydrazone with iodides 2 followed by a one-pot deprotection/spirocyclization sequence. This methodology has been applied successfully to the synthesis of 1,7-dioxaspiro[5.5]undecane and 1,6-dioxaspiro[4.5]decane systems.
\end{abstract}

The 1,7-dioxaspiro[5.5]undecane and the 1,6-dioxaspiro[4.5]decane systems are important subunits of natural products from various sources, including insects, microbes, plants, fungi and marine organisms. ${ }^{1}$ In particular, these moieties occur in a large number of biologically active compounds such as polyether ionophores, insect pheromones and antibiotic macrolides. They have also been employed as scaffold in the synthesis of conformationally restrained glycomimetics. ${ }^{2}$

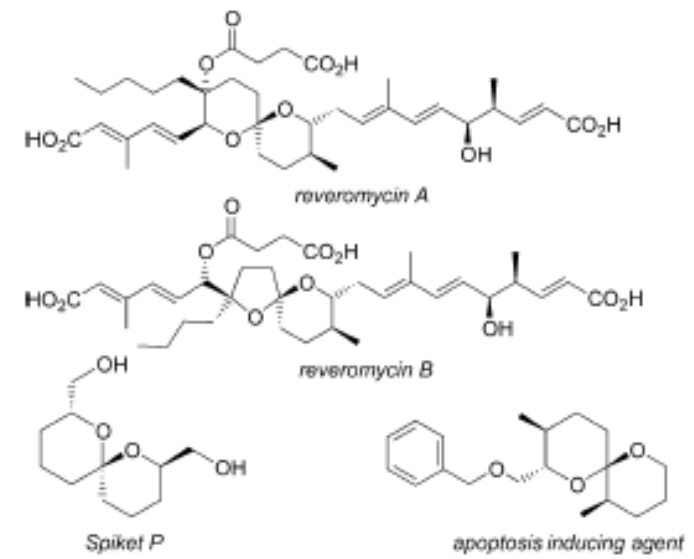

Figure 1.

Keywords: Spiroketals; Stereoselective synthesis; Hydrazone; Isopropylidene iodides.

* Corresponding authors. Tel.: +33 473407 875; fax: +33473407 017; e-mail addresses: icanet@chimie.univ-bpclermont.fr; sinibald@ chimie.univ-bpckermont.fr
As a part of our research programme is devoted to the synthesis of novel antimitotic spiroketal derivatives, we focused our attention on the epidermal growth factor inhibitors reveromycins $\mathrm{A}$ and $\mathrm{B}^{3}$ In contrast with other natural antitumour compounds bearing a spiroketal framework in their skeleton (i.e., spongistatin) these products show a more simplified structure while maintaining interesting activity. Additionally, it was recently reported that poorly substituted spiroketals exhibit biological effects such as tubulin modulation ${ }^{4}$ (Spiket P) and cytotoxicity against tumour cell lines ${ }^{5}$ (Fig. 1).

Therefore, in order to prepare analogues (modulations of sizes and substituents) of spiroketal units, we developed a short and versatile synthesis of frameworks 1 . The key step of our approach is based upon an acidic spirocyclization of a chiral diketalketone $\mathbf{4}$ obtained in three steps from $N, N$-dimethylhydrazones and isopropylidene iodides 2 (Scheme 1).

To check the validity of our approach, we first addressed the synthesis of unsubstituted spiroketals 1 (Scheme 1, $\mathrm{R}=\mathrm{H})$.

First part of our work was devoted to the preparation of synthons $\mathbf{2} \mathbf{a}$ and $\mathbf{2 b}$. Iodide $\mathbf{2 a}$ was synthesized from L-malic acid using an improved sequence (Scheme 2), inspired from the procedure previously described by Mori and Watanabe. ${ }^{6}$ Thus, 2a was obtained in six steps in $47 \%$ overall yield. Compound 2 b was prepared in two steps from commercially available $(S)$-solketal. ${ }^{7}$

Alkylation of the lithiated acetone $N, N$-dimethylhydrazone $^{8}$ with $(S)$-iodide 2 a provided, nearly quantitatively, 


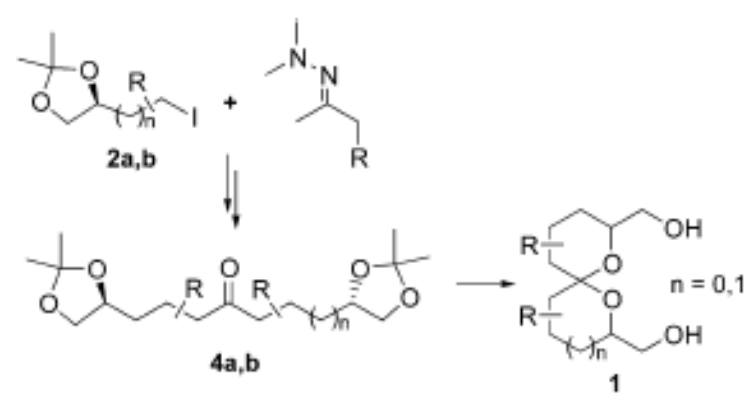

Scheme 1.

the monoalkylated hydrazone 3 , which was immediately used in the next step without further purification. A second alkylation using either iodide $(S)-\mathbf{2} \mathbf{a}$, or $(R)-\mathbf{2} \mathbf{b}$ led, after $\mathrm{SiO}_{2}$-induced cleavage ${ }^{9}$ of the hydrazone function, to the appropriate ketones $4 \mathbf{a}, \mathbf{b}^{10}$ in $43 \%$ and $64 \%$ overall yield, respectively (Scheme 3 ).

Deprotection of the two acetal groups concomitant with spirocyclization was achieved by simple treatment of 4a,b with Amberlyst ${ }^{\infty} 15$ in $\mathrm{MeOH}$ at room temperature during $48 \mathrm{~h}$ (Scheme 3).

In this way, the $(2 S, 6 S, 8 S)-1$ a isomer ${ }^{10}$ was efficiently obtained from 4a as the sole product. The absolute configuration at the central spirocarbon of $1 \mathbf{a}$ is controlled by steric and anomeric effects ${ }^{11}$ whereas the configurations of the carbons bearing the side chains resulted from the configuration of the iodide precursor $\mathbf{2 a}$.

Finally, compound 1a is obtained, without racemization, from $\mathbf{L}$-malic acid in 10 steps and $15 \%$ overall yield. Our methodology is therefore competitive with that reported by Uckun et al. ${ }^{4 a}$ ( 10 steps, $8.6 \%$ yield) or Chattopadhyay and co-workers ${ }^{4 d}$ (eight steps, $15.5 \%$ yield).

In the case of compound $4 b$ the acidic tandem deprotection/cyclization reaction provided, as expected, ${ }^{12}$ a mixture of 1,7-dioxaspiro[5.5] undecane 1b (Scheme 3, pathway a) and 1,6-dioxaspiro[4.5]decanes 1c and 1d (Scheme 3, pathway b), in 93\% yield. Although compound $1 d^{13}$ could be isolated in $9 \%$ yield, compounds

1b and 1e were obtained, in $84 \%$ yield, as an inseparable mixture of isomers ( $3 / 2$ ratio determined from quantitative ${ }^{13} \mathrm{C}$ NMR spectrum). This mixture was then treated with TBDPSCl in DMF in the presence of imidazole, furnishing silylated derivatives $\mathbf{1 e}$ and $\mathbf{1 f}$, which could be separated by flash chromatography. Transformation
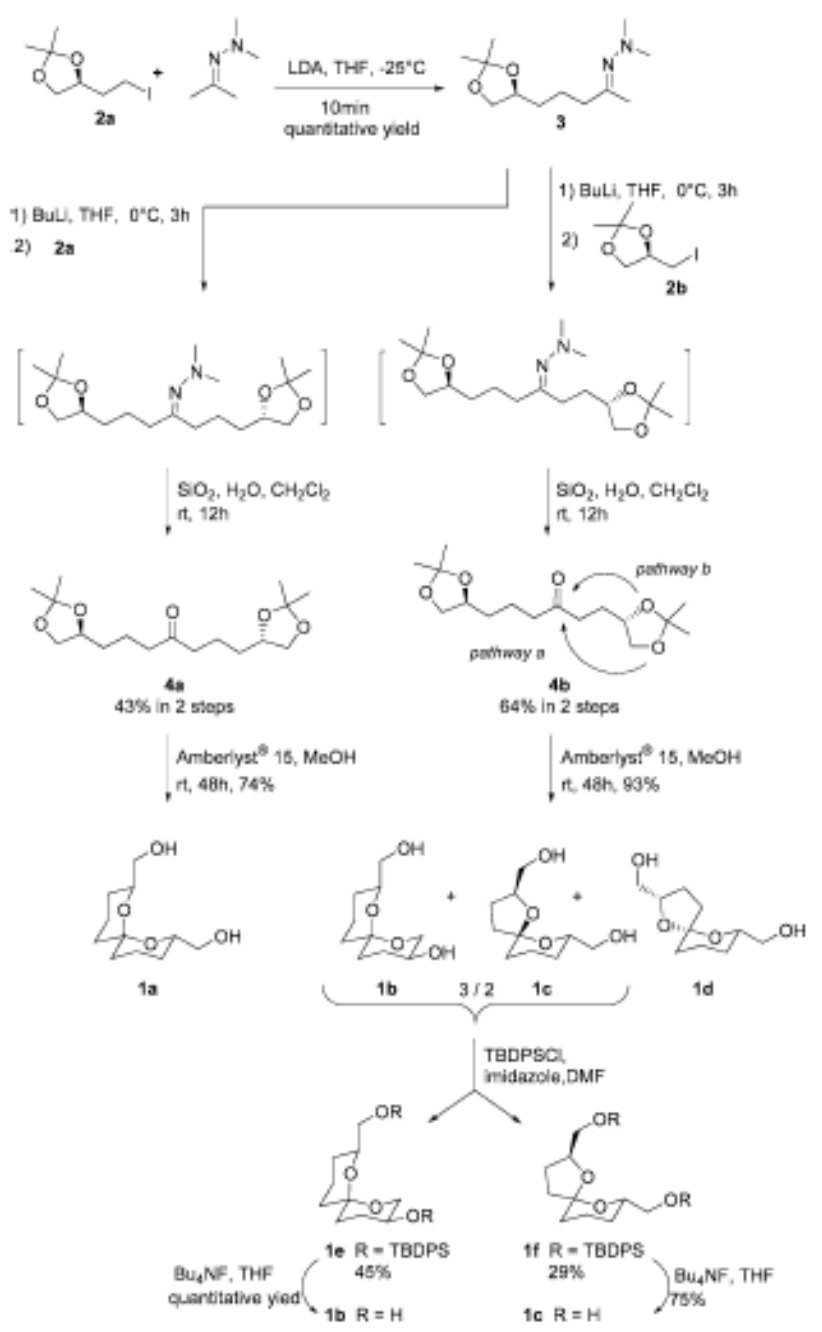

Scheme 3.

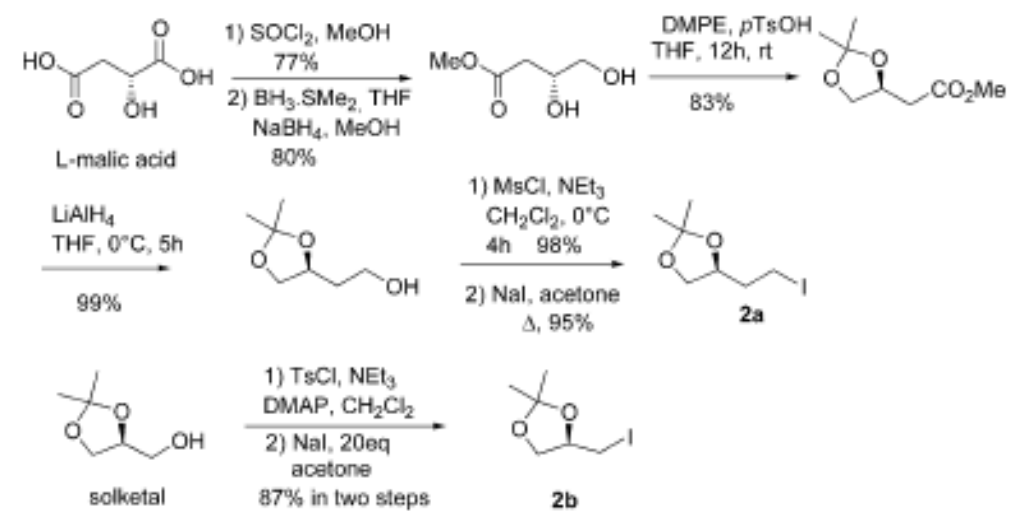

Scheme 2. 


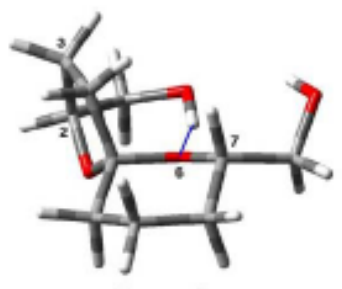

Isomer I

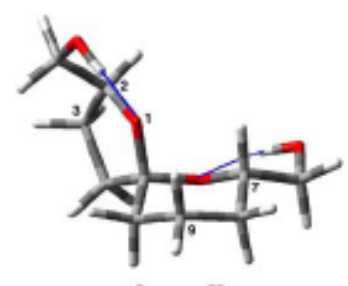

Isomer II
Figure 2. Calculated structures for $(2 S, 5 R, 7 S)$ - and $(2 S, 5 S, 7 S)-1,6$ dioxaspiro[4.5]decanes. Hydrogen bonds are represented by a line.

of compounds le and $1 \mathrm{f}$ to the corresponding alcohols $1 \mathrm{~b}^{13}$ and $1 \mathrm{c}^{13}$ was achieved by a classical method using $\mathrm{Bu}_{4} \mathrm{NF}$ in THF (Scheme 3).

For the same reasons as 1a, the configuration of compound $1 \mathbf{b}$ is $(3 S, 6 S, 8 S)$ as confirmed by the equatorial position of the hydroxyl group at $\mathrm{C}_{3}\left(\mathrm{H}_{3}\right.$ gave a triplet of triplet, $J=10.0$ and $4.5 \mathrm{~Hz}$ ). ${ }^{14}$ We assumed that by taking into account the factors that determine the stereochemistry of 1a the major isomer 1c has configuration $(2 S, 5 S, 7 S)$. In order to confirm this hypothesis, we compared the experimental data to those obtained by molecular modeling (Fig. 2). ${ }^{15}$

The isomer I of $(2 S, 5 R, 7 S)$ configuration (highest energy, $\Delta H_{\mathrm{f}}^{0}=-189.57 \mathrm{kcal} \mathrm{mol}^{-1}$ ) presented a structure in which the lost of one anomeric effect is counterbalanced by an intercyclic hydrogen bond (calculated distance between $\mathrm{OH}$ and $\left.\mathrm{O}_{6}: 1.82 \mathrm{~A}\right)$. The isomer II of $(2 S, 5 S, 7 S)$ configuration, is the more stable isomer $\left(\Delta H_{\mathrm{f}}^{0}=\right.$ $-193.42 \mathrm{kcal} \mathrm{mol}^{-1}$ ) exhibiting the attempted double anomeric structure (Fig. 2). In this isomer, the existence of an 1,3-diaxial relationship between $\mathrm{H}_{7}, \mathrm{H}_{9 \mathrm{xax}}$ and $\mathrm{O}_{1}$ should lead to a deshielded position of the resonances for these two hydrogens. Both calculated structures were in close agreement with the experimental NMR data ${ }^{13}$ as illustrated by (i) the chemical shifts observed for $\mathrm{H}_{7}$ and $\mathrm{H}_{9}$ (1c: $\delta_{\mathbf{H} 7}=3.91 \mathrm{ppm}, \delta_{\mathbf{H} 9 \mathrm{ax}}=1.82 \mathrm{ppm}$, $\delta_{\mathbf{H} 9 \mathrm{eq}}=1.70 \mathrm{ppm} ; 1 \mathbf{1 d :} \delta_{\mathbf{H} 7}=3.70 \mathrm{ppm}, \delta_{\mathbf{H} 9}=1.52$ and $1.31 \mathrm{ppm}$ ) supporting a trans-configured tetrahydropyran ring for 1c, (ii) the calculated and measured scalar coupling constants (Table 1).

In summary, we have developed an efficient and stereoselective approach to 1,7-dioxaspiro[5.5] undecane and to 1,6-dioxaspiro[4.5]decane ligands $\mathbf{1} \mathbf{a}, \mathbf{b}, \mathbf{c}, \mathbf{d}$ from read-

Table 1. Selected calculated and observed scalar coupling constants for $1 \mathrm{c}$ and $1 \mathrm{~d}$

\begin{tabular}{|c|c|c|c|c|}
\hline & & $\begin{array}{l}\text { Calculated } \\
\text { dihedral } \\
\text { angle (deg) }\end{array}$ & $\begin{array}{l}\text { Calculated } \\
J(\mathrm{~Hz})\end{array}$ & $\begin{array}{l}\text { Observed } \\
J(\mathrm{~Hz})\end{array}$ \\
\hline Isomer I (1d) & $\begin{array}{l}\mathrm{H}_{2}-\mathrm{H}_{3 a} \\
\mathrm{H}_{2}-\mathrm{H}_{3 b}\end{array}$ & $\begin{array}{r}1.7 \\
120.4\end{array}$ & $\begin{array}{l}9.6 \\
4.7\end{array}$ & $\begin{array}{l}8.0 \\
2.5\end{array}$ \\
\hline Isomer II (1c) & $\begin{array}{l}\mathrm{H}_{2}-\mathrm{H}_{3 \mathrm{a}} \\
\mathrm{H}_{2}-\mathrm{H}_{3 b} \\
\mathrm{H}_{7}-\mathrm{H}_{8 \mathrm{a}} \\
\mathrm{H}_{7}-\mathrm{H}_{8 \mathrm{~b}}\end{array}$ & $\begin{array}{r}6.7 \\
125.2 \\
172.3 \\
55.1\end{array}$ & $\begin{array}{r}9.5 \\
5.5 \\
12.2 \\
3.9\end{array}$ & $\begin{array}{r}8.0 \\
5.0 \\
12.0 \\
3.0\end{array}$ \\
\hline
\end{tabular}

ily available starting materials. By this route, $\mathbf{1 a}, \mathbf{1 b}, \mathbf{1 c}$ and $1 \mathbf{d}$ were obtained, from iodides $\mathbf{2} \mathbf{a}$ and $\mathbf{2 b}$, in $32 \%$ (four steps), 29\% (six steps), 14\% (six steps) and 6\% (six steps) overall yield, respectively. Furthermore, as the enantiomers of the starting materials are readily available, this approach is also applicable to the synthesis of the antipode spiroketals.

Application of this methodology to the synthesis of substituted spiroketals from modified iodide derivatives 2 is actually in progress in our laboratory. In addition, the antitumoural activity of all synthesized spiroketals will be evaluated in due course.

\section{References and notes}

1. (a) Vaillancourt, V.; Pratt, N. E.; Perron, F.; Albizali, K. F. In The Total Synthesis of Natural Prodacts, John Wiley \& Sons: New York, 1992; Vol. 8; (b) Perron, F.; Albizati, K. F. Chem. Rev. 1989, 89, 1617-1661; (c) Mead, K. T.; Brewer, B. N. Curr. Org. Chem. 2003, 7, 227-256.

2. Bell, W.; Block, M. H.; Cook, C.; Grant, J. A.; Timms, D. J. Chem. Soc., Perkin Trans. 1 1997, 18, 2789-2801.

3. (a) El Sous, M.; Ganame, D.; Tregloan, P. A.; Rizzacasa, M. A. Org. Lett. 2004, 6, 3001-3004; (b) Shimizu, T.; Usui, T.; Machida, K.; Furuya, K.; Osada, H.; Nakata, T. Bioorg. Med. Chem. Lett. 2002, 12, 3363-3366; (c) Tanaka, Y.; Ishikawa, F.; Osada, H.; Imajoh-Ohmi, S.; Uchida, T.; Kakiuchi, T. J. Antibiot. 2002, 55, $904-913$; (d) Cuzzuppe, A. N.; Hutton, C. A.; Lilly, M. J.; Mann, R. K.; McRae, K. J.; Zammit, S. C.; Rizzacasa, M. A. J. Org. Chem. 2001, 66, 2382-2393.

4. (a) Synthesis of spiroketal, named Spiket P by Uckun et al., was pioneered in our laboratory by Jeminet et al. Uckun, F. M.; Mao, C.; Vassilev, A. O.; Huang, H.; Jan, S.-T. Bioorg. Med. Chem. Lett. 2000, 10, 541-545; (b) Huang, H.; Mao, C.; Jan, S.-T.; Uckun, F. M. Tetrahedron Lett. 2000, 41, 1699-1702; (c) Sauret, S.; Cuer, A.; Gourcy, J.-G.; Jeminet, G. Tetrahedron: Asymmetry 1995, 6, 1995-2000; (d) Sharma, A.; Iyer, P.; Gamre, S.; Chattopadhyay, S. Synthesis 2004, 1037-1040.

5. (a) Mitsuhashi, S.; Shima, H.; Kawamura, T.; Kikuchi, K.; Oikawa, M.; Ichihara, A.; Oikawa, H. Bioorg. Med. Chem. Lett. 1999, 9, 2007-2012; (b) Kikuchi, K.; Shima, H.; Mitsuhashi, S.; Suzuki, M.; Oikawa, H. Drugs Future 2000, 25, 501-507.

6. Selected data for compound 2a: ${ }^{1} \mathrm{H}$ NMR $(400 \mathrm{MHz}$, $\mathrm{CDCl}_{3}$ ): $\delta 4.18$ (tdd, $1 \mathrm{H}, J=6.0,7.5,4.5 \mathrm{~Hz}$ ), 4.08 (dd, $1 \mathrm{H}, J=8.0,6.0 \mathrm{~Hz}), 3.57(\mathrm{dd}, 1 \mathrm{H}, J=8.0,6.0 \mathrm{~Hz}), 3.27$ (ddd, $1 \mathrm{H}, J=10.0,5.5,8.0 \mathrm{~Hz}$ ), $3.22(\mathrm{dt}, 1 \mathrm{H}, J=10.0$, $7.5 \mathrm{~Hz}$ ), 2.10 (tdd, $1 \mathrm{H}, J=7.5,14.0,5.5 \mathrm{~Hz}$ ), 2.03 (tdd, $1 \mathrm{H}, J=8.0,14.0,4.5 \mathrm{~Hz}), 1.41(3 \mathrm{H}, \mathrm{s}), 1.35(3 \mathrm{H}, \mathrm{s}) ;{ }^{13} \mathrm{C}$ NMR $\left(100 \mathrm{MHz}, \mathrm{CDCl}_{3}\right): \delta 109.1,75.6,68.6,37.8,26.9$, $25.5,1.2 ;[\alpha]_{\mathrm{D}}^{25}-23.8\left(c 2.1, \mathrm{CHCl}_{3}\right)$ lit. $[\alpha]_{\mathrm{D}}^{25}-22.3(c 2.12$, $\mathrm{CHCl}_{3}$ ) Mori, K.; Watanabe, H. Tetrahedron Lett. 1986, 42, 295-304.

7. Kawakami, Y.; Asai, T.; Umeyama, K.; Yamashita, Y. J. Org. Chem. 1982, 47, 3581-3585.

8. (a) Enders, D.; Dahmen, W.; Dederichs, E.; Gatzweiler, W.; Weuster, P. Synthesis 1990, 1013-1019, (b) Crimmins, M. T.; Rafferty, S. W. Tetrahedon Lett. 1996, 37, 56495652 .

9. Under the used experimental conditions (Kotsuki, H.; Miyazaki, I.; Kadota, M. O. J. Chem. Soc., Perkin Trans. 1 1990, 429-430), no deprotection of the cyclic ketals was detected. 
10. Compounds $4 \mathrm{a}, 4 \mathrm{~b}$ and 1 were characterized by ${ }^{1} \mathrm{H}$ and ${ }^{13} \mathrm{C}$ NMR measurements and mass spectra. In the case of 1a comparison with an authentical sample of our laboratory was also realized. All the data of 1a were in good agreement with the proposed structure 1a: ${ }^{1} \mathrm{H}$ NMR (400 MHz, $\mathrm{CDCl}_{3}$ ): $\delta 3.74$ (dddd, $2 \mathrm{H}, J=11.5$, $7.0,3.0,2.5 \mathrm{~Hz}$ ), 3.60 (dd, $2 \mathrm{H}, J=11.0,3.0 \mathrm{~Hz}), 3.50(\mathrm{dd}$, $2 \mathrm{H}, J=11.0,7.0 \mathrm{~Hz}), 2.20(2 \mathrm{H}, \mathrm{OH}), 1.89(\mathrm{qt}, 2 \mathrm{H}$, $J=13.0,4.5 \mathrm{~Hz}), \quad 1.62 \quad(\mathrm{~m}, 4 \mathrm{H}), 1.50 \quad(\mathrm{md}, 2 \mathrm{H}$, $J=13.0 \mathrm{~Hz}$ ), 1.41 (td, $2 \mathrm{H}, J=13.0,4.5 \mathrm{~Hz}), 1.30$ (tdd, $2 \mathrm{H}, \quad J=13.0,11.5,4.0 \mathrm{~Hz}) ;{ }^{13} \mathrm{C}$ NMR $(100 \mathrm{MHz}$, $\left.\mathrm{CDCl}_{3}\right): \delta 96.3(\mathrm{C} 6), 70.0(\mathrm{C} 2$ and $\mathrm{C} 8), 66.5\left(\mathrm{CH}_{2} \mathrm{OH}\right)$, 35.5 ( $\mathrm{C} 5$ and $\mathrm{C} 11), 26.6$ (C3 and $\mathrm{C} 9), 18.5$ (C4 and $\mathrm{C} 10$ ); $[\alpha]_{\mathrm{D}}^{25}+70.0\left(c 0.46, \mathrm{CHCl}_{3}\right)$. For $(2 R, 6 R, 8 R)-\mathrm{Spi}-$ ket $\mathrm{P}^{4 \mathrm{a}}[\alpha]_{\mathrm{D}}^{22}-59.4\left(c 0.7, \mathrm{CHCl}_{3}\right)$.

11. Deslongchamps, P. Stereoelectronic Effects in Organic Chemistry; Pergamon: New York, 1983; Chapter 2.

12. Mori, K.; Watanabe, H.; Yanagi, K.; Minobe, M. Tetrahedron 1985, 41, 3663-3672.

13. Compound 1b: ${ }^{1} \mathrm{H}$ NMR $\left(400 \mathrm{MHz}, \mathrm{C}_{6} \mathrm{D}_{6}\right): \delta 4.1$ (br s, $2 \mathrm{H}, \mathrm{OH}), 3.76$ (ddd, $1 \mathrm{H}, J=10.0,4.5,2.0 \mathrm{~Hz}), 3.71(\mathrm{~m}$, $1 \mathrm{H}), 3.66(\mathrm{tt}, 1 \mathrm{H}, J=10.0,4.5 \mathrm{~Hz}), 3.57(\mathrm{t}, 1 \mathrm{H}$, $J=10.0 \mathrm{~Hz}), 3.51(\mathrm{dd}, 1 \mathrm{H}, J=11.5,6.5 \mathrm{~Hz}), 3.48(\mathrm{dd}$, $1 \mathrm{H}, J=11.5,4.0 \mathrm{~Hz}), 2.0(\mathrm{~m}, 1 \mathrm{H}), 1.88$ (qt, $1 \mathrm{H}, J=13.0$, $4.0 \mathrm{~Hz}), 1.77(\mathrm{~m}, 1 \mathrm{H}), 1.65(\mathrm{dt}, 1 \mathrm{H}, J=13.0,3.5 \mathrm{~Hz}), 1.46$ (d, $1 \mathrm{H}, J=13 \mathrm{~Hz}), 1.37(\mathrm{~m}, 1 \mathrm{H}), 1.37$ (td, $1 \mathrm{H}, J=13.0$, $4.0 \mathrm{~Hz}), 1.22(\mathrm{~m}, 2 \mathrm{H}), 1.05(\mathrm{qd}, 1 \mathrm{H}, J=12.5,4.0 \mathrm{~Hz}) ;{ }^{13} \mathrm{C}$ NMR $\left(100 \mathrm{MHz}, \mathrm{C}_{6} \mathrm{D}_{6}\right): \delta 95.0(\mathrm{C} 6), 71.0(\mathrm{C} 2), 66.4$ $\left(\mathrm{CH}_{2} \mathrm{OH}\right), 66.3$ (C9), 64.8 (C8), 35.2 (C11), 34.6 (C5), 28.3 (C10), 26.8 (C3), 19.0 (C4); IR (film) 3401, 2944, 1660, $1446,1377,1227,1177,1054,1018 ;[\alpha]_{\mathrm{D}}^{25}+76.43(c 1.26$, $\left.\mathrm{CHCl}_{3}\right)$. Compound 1c: ${ }^{1} \mathrm{H}$ NMR $\left(400 \mathrm{MHz}, \mathrm{CDCl}_{3}\right): \delta$ 4.21 (dtd, $1 \mathrm{H}, J=8.0,5.5,3.0 \mathrm{~Hz}$ ), 3.91 (ddt, $1 \mathrm{H}, J=11.5$,
$6.5,3.0 \mathrm{~Hz}$ ), 3.73 (dd, $1 \mathrm{H}, J=11.5,3.0 \mathrm{~Hz}), 3.58(\mathrm{dd}, 1 \mathrm{H}$, $J=11.5,3.0 \mathrm{~Hz}), 3.52(\mathrm{dd}, 1 \mathrm{H}, J=11.5,6.0 \mathrm{~Hz}), 3.48(\mathrm{dd}$, $1 \mathrm{H}, J=11.5,6.5 \mathrm{~Hz}), 2.3(\mathrm{br} \mathrm{s}, 2 \mathrm{H}, \mathrm{OH}), 2.1(\mathrm{~m}, 1 \mathrm{H}), 1.96$ $(\mathrm{m}, 1 \mathrm{H}), 1.82(\mathrm{qt}, 1 \mathrm{H}, J=13.0,4.0 \mathrm{~Hz}), 1.70(\mathrm{~m}, 5 \mathrm{H}), 1.50$ (dq, $1 \mathrm{H}, J=13.0,3.0 \mathrm{~Hz}$ ), 1.32 (qd, $1 \mathrm{H}, J=13.0,3.5 \mathrm{~Hz}$ ); ${ }^{13} \mathrm{C}$ NMR $\left(100 \mathrm{MHz}, \mathrm{CDCl}_{3}\right): \delta 106.6(\mathrm{C} 5), 78.2(\mathrm{C} 2)$, $71.0(\mathrm{C} 7), 66.0\left(\mathrm{CH}_{2} \mathrm{OH}\right), 64.7\left(\mathrm{CH}_{2} \mathrm{OH}\right), 37.7(\mathrm{C} 10), 32.9$ (C4), 26.2 (C8), 25.3 (C3), 19.6 (C9); IR (film) 3335, 2941, $1150,1380,1222,1042 ;[\alpha]^{25}+70.87\left(c 1.38, \mathrm{CHCl}_{3}\right)$. Compound 1d: ${ }^{1} \mathrm{H}$ NMR (400 MHz, $\left.\mathrm{CDCl}_{3}\right): \delta 4.36$ (tt, $1 \mathrm{H}, J=8.0,2.5 \mathrm{~Hz}), 3.84(\mathrm{dd}, 1 \mathrm{H}, J=12.0,2.5 \mathrm{~Hz}), 3.70$ $(\mathrm{m}, 1 \mathrm{H}), 3.65(\mathrm{br} \mathrm{s}, 2 \mathrm{H}, \mathrm{OH}), 3.62(\mathrm{~m}, 2 \mathrm{H}), 3.48(\mathrm{dd}, 1 \mathrm{H}$, $J=12.0,8.0 \mathrm{~Hz}), 2.4(\mathrm{~m}, 1 \mathrm{H}), 2.1(\mathrm{~m}, 1 \mathrm{H}), 1.90(\mathrm{~m}, 2 \mathrm{H})$, $1.74(\mathrm{~m}, 1 \mathrm{H}), 1.62(\mathrm{~m}, 2 \mathrm{H}), 1.52(\mathrm{~m}, 2 \mathrm{H}), 1.31(\mathrm{~m}, 1 \mathrm{H}) ;{ }^{13} \mathrm{C}$ NMR $\left(100 \mathrm{MHz}, \mathrm{CDCl}_{3}\right): \delta 107.6(\mathrm{C} 5), 81.2(\mathrm{C} 7), 75.6$ (C2), $66.3\left(\mathrm{CH}_{2} \mathrm{OH}\right), 64.0\left(\mathrm{CH}_{2} \mathrm{OH}\right), 34.0(\mathrm{C} 4), 33.3(\mathrm{C} 6)$, 26.2 (C3), 24.2 (C8), 21.1 (C9); $[\alpha]_{\mathrm{D}}^{25}+18.84$ (c 0.34 , $\mathrm{CHCl}_{3}$.

14. Baker, R.; Herbert, R. H. J. Chem. Soc., Perkin Trans 1 1987, 1123-1127.

15. Conformational analysis was performed using MonteCarlo multiple method (Chang, G.; Wayne, C.; Guida, W. C.; Still, W. C. J. Am. Chem. Soc. 1989, 111, 4379-4386) with MM3 force field (Allinger, N. L.; Yuh, Y. H.; Lii, J. H. J. Am. Chem. Soc. 1989, 111, 8551-8566) of Macromodel 7.0 program (Macromodel 7.0, Shroedinger Inc., 1500 SW First Ave., Suite 1180, Portland, OR 97201, USA). Then quantum semi-empirical calculations SAM1 (Dewar, M. J. S.; Lie, C.; Yu, J. Tetrahedron 1993, 49, 5003-5038) on both isomers global minima geometries found previously were carried out using AMPAC 7.0 program (Ampac7.0, 2000 Semichen, 7128 Summit, Shawnee, KS 66216, USA). 